\title{
The super-quadratic growth of high- harmonic signal as a function of pressure
}

To the Editor - A super-quadratic growth of high-harmonic (HHG) signal was measured by Seres et al. ${ }^{1}$ over more than five orders of magnitude as a function of gas pressure and for a large range of laser intensities. The authors explain this impressive growth as the signature of a strong increase of the extreme ultraviolet (XUV) signal induced by an additional stimulated effect, which they call X-ray parametric amplification (XPA). We performed a detailed analysis of the results in ref. 1 using the theory of pure HHG and taking into account all the effects of phase-matching. Our results demonstrate that, without introducing any adjustable parameter, phase-matching can account for the super-quadratic growth, and that pure HHG yields are in good agreement with the experimental data of ref. 1. Semiclassical ${ }^{2,3}$ and quantum ${ }^{4}$ theories provide accurate estimates of the initial amplitude and phase of the XUV polarization generated during single atom and electron interaction in the HHG processes.

Phase-matching macroscopic conditions are fully described to study the coherence between the XUV and the infrared fields along the generative medium, and a coherence length is calculated ${ }^{5}$. The final XUV intensity strongly depends on the value of the coherence length, influenced by the atomic and electronic dispersions, the Gouy phase and the atomic phase gradients ${ }^{6}$. These effects were included in the numerical code described in ref. 6 and ran for the conditions of ref. 1 . An example of the results obtained for the 43rd harmonic is shown in Fig. 1, where a strong exponential growth at low pressure is clearly visible, before saturation takes place at higher pressures, in good agreement with the data of ref. 1. The super-quadratic behaviour was observed over a large set of harmonic orders and laser intensities from 1 to $2 \times 10^{14} \mathrm{~W} \mathrm{~cm}^{-2}$. The relatively low laser intensity and short focusing used in ref. 1, as compared with more usual conditions with higher efficiency such as in refs 8 and 9 , lead to a strong increase of the coherence length with pressure by compensation of the Gouy phase gradient through atomic dispersion in neutral gas,

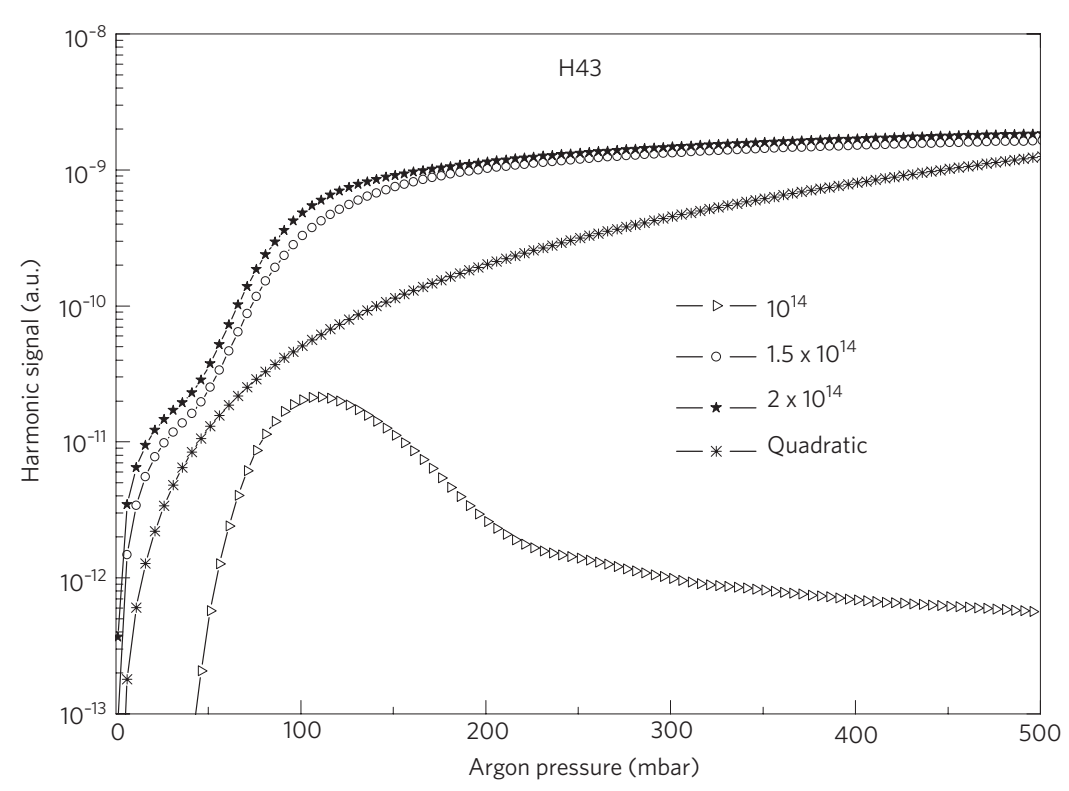

Figure 1 | Signal from $\mathrm{H} 43$ as a function of pressure for three laser intensities, 1, 1.5 and $2 \times 10^{14} \mathrm{~W} \mathrm{~cm}{ }^{-2}$, compared with quadratic behaviour. a.u., arbitrary units.

already described in ref. 7. This coherence length increase comes in addition to the quadratic growth of the atomic response with pressure to produce the superquadratic behaviour. We consequently explain why this effect is not observed at looser focusing with smaller Gouy phase gradient. Moreover, we found that in the experimental conditions of ref. 1 , the atomic phase gradient from the long trajectory is more favourable than the short one to increase the poor phasematching conditions at low pressures and low intensities, and thus low signal, which can explain why both the spectral and spatial profiles are much wider in those conditions than at optimum pressures when the short trajectory dominates. As it is well known that the atomic phase spectral broadening is red for the rising front and blue for the rear one, global redshifting means that harmonics are mostly generated on the rising front. Our model also explains the signal inflexion at low pressure and low intensity in terms of temporal phase-matching fringes. In conclusion, our simulations show that the experimental data of ref. 1 do not definitely demonstrate any additional effect such as stimulated or parametric amplification rather than peculiar properties of pure high harmonic emission.

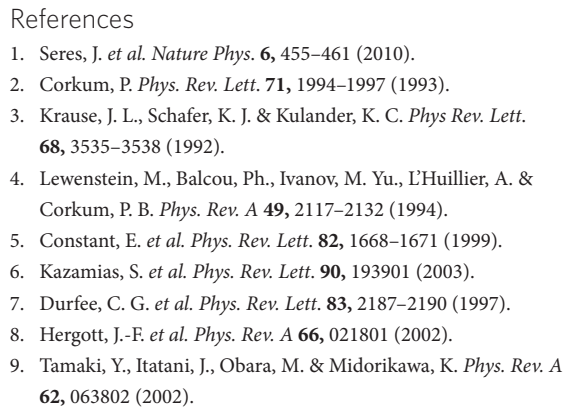

S. Kazamias ${ }^{1,2,3 *}$, S. Daboussi ${ }^{1,2,3}$,

O. Guilbaud1,2,3, K. Cassou 1,2,3, C. Montet ${ }^{1,2,3}$,

O. Neveu ${ }^{1,2,3}$, B. $\operatorname{Cros}^{1,2,3}$, D. Ros ${ }^{1,2,3}$ and G. Maynard,2

1 University Paris-Sud, Laboratoire de Physique des Gaz et des Plasmas, UMR 8578, Orsay F-91405, France, ${ }^{2}$ CNRS, Orsay F-91405,

France, ${ }^{2}$ LASERIX, CLUPS, University Paris-Sud, Orsay F-91405, France

^e-mail: sophie.kazamias@u-psud.fr 